\title{
Analysis of the Responses of Metal-Oxide Semiconductor Nanostructures to Nitrogen Dioxide
}

\begin{abstract}
M. ProceK* And T. Pustelny
Department of Optoelectronics, Silesian University of Technology, Akademicka 2, 44-100 Gliwice, Poland

The paper concerns analyses of the nanostructures based on metal-oxide semiconductors, such as titanium dioxide $\left(\mathrm{TiO}_{2}\right)$ and zinc oxide $(\mathrm{ZnO})$ exposed to nitrogen dioxide $\left(\mathrm{NO}_{2}\right)$ in an atmosphere of dry and humid synthetic air. The investigations were performed at a rather low temperature amounting to $120^{\circ} \mathrm{C}$. The responses of sensors differing in the morphology of their structures have been compared. Also the time of their responses were compared with each other. The paper describes theoretically changes in the effective resistance of these structures due to the interaction of the gas. The presented results are being discussed based on the analysis of physical and chemical phenomena.
\end{abstract}

DOI: $10.12693 /$ APhysPolA.124.529

PACS: 82.47.Rs, 07.07.Df

\section{Introduction}

While designing gaseous sensor structures it is inevitable to be well acquainted with the physico-chemical phenomena occurring in the course of contacting the absorber with the tested gas. Indispensable is also the knowledge of the physics of these phenomena in the structures and their effect on the formation of the measurement signal. For many years the very popular and well-known structures sensitive to gases have proved to be semiconductor structures. The most popular semiconductors applied as sensors are some metal-oxides, being wide-band gap semiconductors, for instance: $\mathrm{SnO}_{2}$ [1], $\mathrm{TiO}_{2}[2], \mathrm{WO}_{3}[3], \mathrm{ZnO}[4,5], \mathrm{GaP}[6]$, and others. In spite of their considerable popularity and intensive investigations, the phenomena occurring in them during their interaction with gases are still not fully known. No final formalism has been elaborated yet, which would permit to describe definitely the mechanism of the formation of measurement signals in thin smooth and porous layers [7]. In recent years considerable developments have been observed in the field of nanotechnology, thanks to which the researchers have to their disposal better methods of obtaining nano- and microstructures. Various new forms of nano- and microstructures have become available, differing both in their morphology and chemical composition, as well as in the kind of crystalline lattice. Many new nanostructures are being tested from the viewpoint of their application in gas sensors. As an example let us take graphene [8] and oxide graphene [9]. Nanostructures of wide-band gap semiconductors are nowadays tested with purpose of applying them in sensors of gases and vapours $[10,11]$. Vast possibilities of using nanostructures as sensors are due to their extensive surface, the multitude of connections between the crystals and the easiness of their doping by nanoparticles of metals $[12,13]$.

*corresponding author; e-mail: Marcin.Procek@polsl.pl
This paper presents and characterizes nanoparticles of $\mathrm{TiO}_{2}$ and $\mathrm{ZnO}$, which are wide-band gap semiconductors of the type $n$. The investigated structures display a different morphology resulting from differences in means of their synthesizing. Their responses to $\mathrm{NO}_{2}$ in the atmosphere of air have been presented and compared both in dry and humid conditions. The paper deals also with an attempt of analyzing the achieved results from the viewpoint of describing the physicochemical phenomena which are to be observed in these semiconductor structures affected by the interaction with gas. These conditions are based on the physics of thin porous layers. The authors wish to demonstrate the similarities and differences existing between thin layers and the nanoparticles.

\section{Theoretical}

Changes in the conductivity of electricity in semiconductor planar structures are mainly due to changes in the concentration of the carriers. The structure interacts with the gases through the surface of the semiconductor. The gas in the vicinity of the surface can be adsorbed either physically or chemically. The adsorption of gas leads to changes of the concentration of the carriers in semiconductor - an atom or particle of gas may add or remove an electron from the conduction band, and also leads to a formation of traps on the surface $[14,15]$.

Porous layers are supposed to possess a double Schottky barrier between the grains. The electric potential barriers at the connections of the grains, as well as the number of free carriers of the load and their spatial distribution depend on the gas affecting the surface of the semiconductor. Therefore, the electrical conductivity in the planar semiconductor structure is also dependent on the kind and concentration of the given gas $[10,13,14]$. Semiconductor structures operating in a natural environment are exposed to high concentration of oxygen $\mathrm{O}_{2}$ (ca. 21\%). Also humidity is always present in the atmosphere. It is, therefore, of much importance to determine the influence of changes of the concentration of $\mathrm{O}_{2}$, and $\mathrm{H}_{2} \mathrm{O}$ in air on the change of conductivity in 
semiconductor structures. The concentration of oxygen in the atmosphere may be considered as being nearly constant, whereas the humidity of air changes considerably, depending on its temperature, atmospheric precipitations, atmospheric fronts and the vicinity of water regions. There are various ways of the interaction of a semiconductor structure with oxidizing and reductive gases. The latter ones "consume" the oxygen in the structure.

In metal-oxide semiconductors the reducing gases may also interact with the oxygen from the crystal lattice. The results of these interactions are reductions of the potential barrier and electrical resistance. Oxidizing gases are adsorbed on the same active centres, on which oxygen is absorbed, competing with it. If in the planar structure the adsorption of the oxidizing gas exceeds adsorption of oxygen, the potential barrier grows resulting in an increase of the electrical resistance of the structure. The electrons may also tunnel through small gaps between the grains even when the boundaries of the grains are not fully formed (the grains remain close to each other). In such a case the tunneled current is also attenuated by the surface potential of the grains, so that share (contribution) in the change of the electrical resistance is similar to the barrier effect.

Thus, two fundamental ways of the flow of electrical current in the planar structure of a metal-oxide semiconductor are to be distinguished, viz.: through the potential barrier and by tunneling when the grains are not directly in contact to each other $[13,14]$.

Generally, for description of semiconductor planar structure the concept "receptor function" is applied, i.e. the response of a single crystalline grain (crystallite) to the gas acting and "transducer function" characterizing the effect of the interaction of the gas on the connections between the crystallites [14]. Both, $\mathrm{TiO}_{2}$ and $\mathrm{ZnO}$ in their pure form, are semiconductors of the type $n$; therefore, just this type is going to be considered here. These considerations also concern merely oxidizing gases, presented by $\mathrm{NO}_{2}$.

Assuming that radius of semiconductor crystallites exceeds the Debye length, the depletion layer may be approximated as a one-dimensional model (Fig. 1a). The existence of the surface states below the Fermi level causes the transfer of electrons from the conduction band to the surface states until an equilibrium has been attained. The bending of energy bands must in the case of small structures be considered differently. In this case the half-infinite model cannot be applied, because the bendings of the bands from every edge of the crystallite must be taken into account (Fig. 1b) as a new type of depletion [14]. The resulting depletion layer can be described by the depth profile of potential energy of electrons $q V(x)$. Characteristic quantities are in this case the surface barrier of the potential $q V_{s}$, the depth of depletion $w$, and the density of the spatial electrical charge $\rho(x)$. In the case of semiconductors of the type $n$ the density of the spatial charge is $\rho(x)=q\left[N_{d}^{+}(x)-n(x)\right]$, where $N_{d}^{+}(x)$ and $n(x)$ are, re- spectively, the density of the ionized donors and density of conductive electrons at the given depth. When all the donors are ionized, we may assume the model of sudden changes $\rho(x)$ :

$$
\begin{aligned}
& \rho(x)=q N_{d} \text { for } 0<x<w, \\
& \rho(x)=0 \text { for } w<x, \\
& Q_{\mathrm{sc}}=-q N_{d} w
\end{aligned}
$$

where $N_{d}$ - density of the donors, treated as a constant in the volume of oxide; $Q_{\mathrm{sc}}$ - surface density of the electric charge.

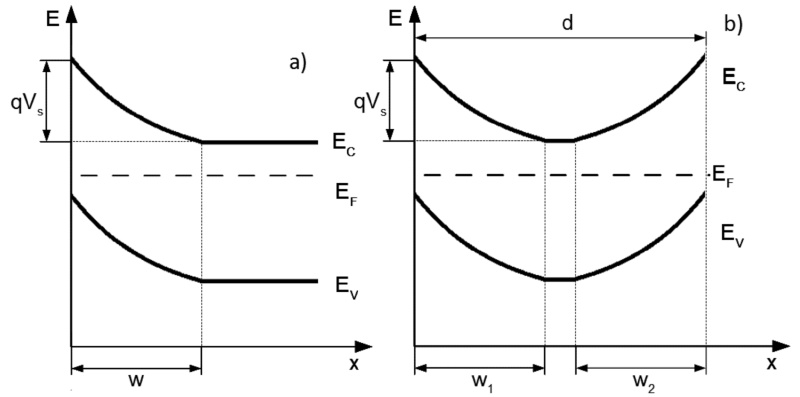

Fig. 1. Simplified energy model of the planar wide-bandgap semiconductor interacting with gas: (a) in the half-infinite case; (b) concerning a particle of minute dimension ( $w$ - the depth of the zone of depletion, $d$ - the size of the nanostructure, $q V$ - the height of potential barrier).

By solving Poisson's equation for near surface region in semiconductor we get the expression for $q V(x)$ as

$$
q V(x)=\left[q^{2} N_{d} /(2 \varepsilon)\right](x-w)^{2} .
$$

Hence it becomes obvious that the height of the potential barrier depends to a large extent on the depth of depletion, which again depends directly on the amount of oxygen adsorbed on the surface of the structure or some other oxidizing gas, e.g. $\mathrm{NO}_{2}$. The influence of the depth of depletion on nanoparticles is still greater (in which case the half-infinite model cannot be applied).

At temperature below $420 \mathrm{~K}$ in dry condition oxygen molecules are absorbed on metal oxides mainly physically by interaction of Van der Waals's type; there may also occur chemisorptions in the form of molecule ions $\mathrm{O}_{2}^{-}$. Within the temperature range from $420 \mathrm{~K}$ to $670 \mathrm{~K}$ the chemisorptions of $\mathrm{O}^{-}$ions take place. Above $670 \mathrm{~K}$ both $\mathrm{O}^{-}$and $\mathrm{O}^{2-}$ ions occur $[11,12]$. $\mathrm{NO}_{2}$ molecules can be adsorbed either physically as neutral particles or chemically as $\mathrm{NO}_{2}^{-}$ions, competing with oxygen for reaching the active centres, up to the achievement of an equilibrium in this process.

Important are also processes of the diffusion of gas molecules into the semiconductor layer. $\mathrm{NO}_{2}$ molecules are rather big particles, due to which the diffusion of this gas into the structure as a solid layer or a layer with small pores is rather slow. The application of sensing layer consisting of nanostructures should facilitate the penetration of $\mathrm{NO}_{2}$ of a whole layer and ensure the ap- 
plicability of Knudsen's diffusion model. The electrical conductivity is also affected by the presence of humidity in the gas mixture. At low temperatures the $\mathrm{H}_{2} \mathrm{O}$ molecules are adsorbed physically, whereas elevated temperatures cause a dissociation into $\mathrm{OH}^{-}$and $\mathrm{H}^{+}$ions, subject to chemisorptions. OH groups as well as the hydrogen atoms $\mathrm{H}$ consume oxygen adsorbed on the surface of the semiconductor, in result of which the potential barrier is decreased and the active centres that can adsorb molecules of oxygen or some other oxidizing gas are unloaded [11].

\section{Experimental}

\subsection{Characterization of investigated metal oxide} nanostructures

The method of synthesizing the investigated semiconductor materials and depositing them on the transducer have been dealt with in detail by the authors of [12]; the tested materials being titanium dioxide $\left(\mathrm{TiO}_{2}\right)$ and zinc oxide $(\mathrm{ZnO})$ in the form of nano- and microstructures. Four different structures are to be distinguished, viz.: $\mathrm{TiO}_{2} 350, \mathrm{TiO}_{2} 450, \mathrm{ZnO} \mathrm{A}$ and $\mathrm{ZnO} \mathrm{B}$. The way of synthesizing differed in the temperature of calcinations in the case of $\mathrm{TiO}_{2}\left(350{ }^{\circ} \mathrm{C}\right.$ and $\left.450{ }^{\circ} \mathrm{C}\right)$. In the case of $\mathrm{ZnO}$ the differences consisted in the ratios of the substrates and the time of mixing in ultrasounds. The morphology of the investigated structures can be seen in the images of scanning electron microscopy (SEM) presented in Fig. 2. As we see, $\mathrm{TiO}_{2}$ structures have the form of nanotubes and nanorods, which in both cases are equal to each other and not homogeneous. The size of the tubes differs from tens $\mathrm{nm}$ to several $\mu \mathrm{m}$, and between them impurities may be detected in the form of lumps (solid bodies). Frequently also such lumps consist of glued tubes.

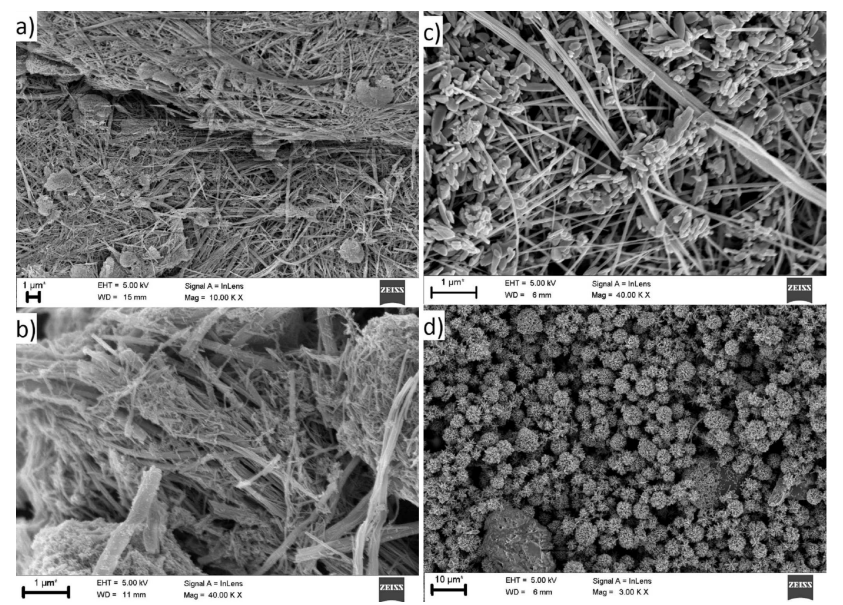

Fig. 2. SEM images of investigated structures: (a) $\mathrm{TiO}_{2} 350$, (b) $\mathrm{TiO}_{2} 450$, (c) $\mathrm{ZnO} \mathrm{A}$, (d) $\mathrm{ZnO} \mathrm{B}$.

In spite of their morphological similarity, $\mathrm{TiO}_{2}$ structures differ in the basic electrical resistance, amounting to about $200 \Omega$ for $\mathrm{TiO}_{2} 350$ and $16 \Omega$ for $\mathrm{TiO}_{2} 450$, respectively. These differences of resistance values may result from differences in the crystalline structure or from the accidental position or differing adhesion of the structure to the substrate.

In the case of $\mathrm{ZnO}$ two differing morphologies have been distinguished, namely non-homogeneous nanotubes polluted by nanolumps ( $\mathrm{ZnO} \mathrm{A}$ ) and agglomerates of nanotubes in the shape of highly homogenic flowers $(\mathrm{ZnO} \mathrm{B})$. Although these structures differ distinctly in their morphology, they display a relatively similar basic resistance, amounting to $17 \Omega$ in the case of $\mathrm{ZnO} \mathrm{A}$ and $19 \Omega$ in the case of $\mathrm{ZnO} \mathrm{B}$.

All the four structures are characterized by an evident random position of the nanoparticles. This may be explained by random and numerous electric connections between the individual nanoparticles and their agglomerates. This is probably the reason for the number of direct connections (merely the potential barrier) and indirect connections (tunneling) or gaps (a lack of electrical conductivity). The effective resistances of all the four structures are, however, surprisingly low with respect to the applied materials. As has already been mentioned, the resistance was measured at $115^{\circ} \mathrm{C}$; hence certainty that humidity must have been removed from the material (in humid presence no considerable changes of the resistance had been observed). Such a low resistance may have been caused by connecting parallel a large number of paths of nanostructures, which may be compared with a parallel positioning of many resistors with a high effective resistance, displaying a low resultant resistance. Also the presence of the crystalline phase predominating the atrophic phase with a high-resistance influences the value of the effective electrical resistance of the structure.

\subsection{Measurement details}

The resistance was measured making use of a four interdigital transducers with electrodes situated at a distance of $20 \mu \mathrm{m}$ from each other. Electrodes of the transducers are made of gold and had a thickness of $100 \mathrm{~nm}$. They were placed on a glass substrate with a thin layer of chromium ( $5 \mathrm{~nm}$ ) between the gold and glass. The respective channels were coated dropwise with absorbers dispersed in ethanol in the form of the $\mathrm{TiO}_{2}$ and $\mathrm{ZnO}$ nanostructures mentioned above. The application of the interdigital transducer permitted to measure all the four structures simultaneously, ensuring identical conditions of measurements and making it possible to compare the obtained results. In all these measurements the atmosphere was synthetic air $\left(20 \% \mathrm{O}_{2}\right.$ and $\left.80 \% \mathrm{~N}_{2}\right)$. The tests were run with a continuous flow of synthetic air at a rate of $500 \mathrm{ml} / \mathrm{min}$. The measuring cycle always comprised 30 min of nitrogen dioxide $\left(\mathrm{NO}_{2}\right)$ with a predetermined concentration in air and $30 \mathrm{~min}$ of pure synthetic air. The measurements were accomplished at two different relative humidities of the gas mixture $\mathrm{RH}=5 \%$ and $\mathrm{RH}=50 \%$ (measured at $22^{\circ} \mathrm{C}$ ). Three concentrations of $\mathrm{NO}_{2}$ were tested, viz. 80,200 , and $400 \mathrm{ppm}$.

The aim of the investigations was to carry out measurements at a relatively low temperature of $115^{\circ} \mathrm{C}$, which is high enough to prevent an accumulation of water in the 
deposit, but also low enough to determine the effect of physical and chemical sorption on the electrical properties of the structure. Such low temperature is also pivotal for the reduction of the energy consumption of future sensors, which can be constructed basing on the investigated structures. The resistance of the respective transducer with a sensor structure was measured by applying the switch unit AGILENT 34970. The accuracy of the applied measurement card amounted to $\Delta R= \pm 50 \times 10^{-6} \Omega$ in the range $R=10-100 \Omega$ and $\Delta R= \pm 0.5 \times 10^{-3} \Omega$ in the range $R=100-999 \Omega$. Every single channel of the sensor was connected to different channels of the card. More details concerning the structures, the operation of the transducer and the measuring stand may be found in $[12,13]$.

\subsection{Experimental results}

The results obtained in the investigations have been presented as differential changes of the resistance

$$
\Delta R=R-R_{0},
$$

where $R_{0}$ denotes the basic resistance of the structure measured in pure synthetic air. Thus it becomes possible to express changes of the resistance in $\mathrm{m} \Omega$. The results concerning every tested structure have been presented for three different concentrations of $\mathrm{NO}_{2}: 80,200$ and $400 \mathrm{ppm}$ in dry and in humid conditions (Fig. 3).

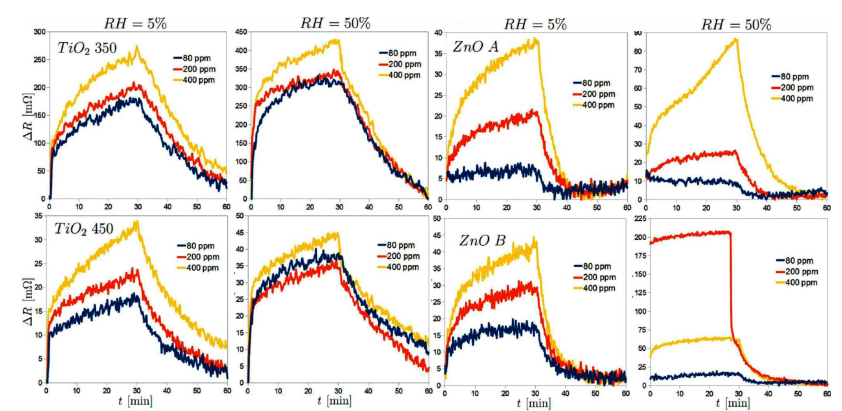

Fig. 3. Response of presented structures on $\mathrm{NO}_{2}$ in dry $(\mathrm{RH}=5 \%)$ and wet $(\mathrm{RH}=50 \%)$ air. Structure temperature $T=115^{\circ} \mathrm{C}$, humidity was measured at $22^{\circ} \mathrm{C}$. First 30 min of air with given concentration of $\mathrm{NO}_{2}$ and then 30 min of clean air was given.

TABLE

The time of responses achieved by the respective sensors $t_{\mathrm{s}}$ and $t_{\mathrm{d}}$ denoting the time of sorption and desorption of gas. (The time of response was assessed as the time which passed from the achievement of $10 \%$ to $90 \%$ of the value of saturation of the signal.)

\begin{tabular}{c|c|c|c|c|c|c|c|c|c}
\hline \hline \multirow{2}{*}{ Structure } & \multirow{2}{*}{$\begin{array}{c}\mathrm{NO}_{2} \text { concen- } \\
\text { tration [ppm] }\end{array}$} & \multicolumn{4}{|c|}{$\mathrm{RH}=5 \%$} & \multicolumn{4}{c}{$\mathrm{RH}=50 \%$} \\
\cline { 3 - 9 } & & 80 & 200 & 400 & Average & 80 & 200 & 400 & Average \\
\hline \multirow{2}{*}{350} & $t_{\mathrm{s}}[\mathrm{min}]$ & 19.8 & 20.3 & 22.3 & 20.8 & 13.8 & 13.1 & 17.1 & 14.7 \\
& $t_{\mathrm{d}}[\mathrm{min}]$ & 25.9 & 28.2 & 28.9 & 27.7 & 24.5 & 24.2 & 25.1 & 24.6 \\
\hline \multirow{2}{*}{$\mathrm{TiO}_{2} 450$} & $t_{\mathrm{s}}[\mathrm{min}]$ & 19.6 & 22.8 & 22.3 & 21.6 & 14.2 & 15.8 & 15.6 & 15.2 \\
& $t_{\mathrm{d}}[\mathrm{min}]$ & 23.5 & 27.8 & 29.0 & 26.8 & 28.8 & 27.5 & 29.8 & 28.7 \\
\hline \multirow{2}{*}{$\mathrm{ZnO} A$} & $t_{\mathrm{s}}[\mathrm{min}]$ & - & 20.0 & 19.8 & 19.9 & - & 14.1 & 25.0 & 19.6 \\
& $t_{\mathrm{d}}[\mathrm{min}]$ & - & 10.5 & 8.7 & 9.6 & - & 8.1 & 15.3 & 11.7 \\
\hline \multirow{2}{*}{$\mathrm{ZnO} \mathrm{B}$} & $t_{\mathrm{s}}[\mathrm{min}]$ & 12.1 & 15.5 & 22.2 & 16.6 & - & - & 7.6 & - \\
& $t_{\mathrm{d}}[\mathrm{min}]$ & 14.0 & 10.0 & 10.0 & 11.3 & - & - & 100 & -
\end{tabular}

As had to be expected, the oxidizing gas $\mathrm{NO}_{2}$ increased the electrical resistance in all cases. Measurements of $\mathrm{ZnO} \mathrm{B}$ at $\mathrm{RH}=50 \%$ and a concentration of $200 \mathrm{ppm}$ permit to understand the problem concerning the electrical contact - this result has been plotted for the purpose of its presentation, without taking it into account in further analyses. The time duration of responses of the structures to the interaction with the gaseous environment has been gathered in Table. As a measure of the time duration of responses the time interval has been assumed which passed from the achievement of $10 \%$ to $90 \%$ of the maximum value of the response at the given concentration, concerning both the sorption of $\mathrm{NO}_{2}$ and its desorption from the structure. These durations have been averaged for the purpose of comparing them with each other.

\section{Discussion and conclusions}

The structures were tested at temperature of $115^{\circ} \mathrm{C}$, which is a comparatively low temperature in the case of the investigated materials (in the technology of sensors the temperature of their operation is in the range from $200{ }^{\circ} \mathrm{C}$ to $500{ }^{\circ} \mathrm{C}$ ). It is worth to know and to compare the responses of sensors in conditions of a low and high humidity in an atmosphere of synthetic air, because those conditions correspond approximately to those of the environment. In the dry gas mixture an increase of the concentration of $\mathrm{NO}_{2}$ involved a proportional increase of 
the value of changes in the resistance of the structure. In the case of a humid mixture the proportionality of $\mathrm{NO}_{2}$ to resistance is not a rule, e.g. for $\mathrm{TiO}_{2} 450$ at $\mathrm{RH}=50 \%$ the time of responses the resistance for $80 \mathrm{ppm}$ exceeds the response for $100 \mathrm{ppm}$. In the case of $\mathrm{TiO}_{2}$ structures the time of responses are similar to each other independently of the temperature of calcinations both during the sorption and desorption of gases when $\mathrm{RH}=5 \%$ (Table). A nearly two times greater change in the resistance of $\mathrm{TiO}_{2} 450$ is due to the basic difference (which proved to be comparatively higher); the times of response of these structures is similar.

As we see, $\mathrm{ZnO}$ structures display in spite of similar basic electrical resistances different thresholds of detectability of $\mathrm{NO}_{2}$, depending on their morphology. In the case of $\mathrm{ZnO}$ no reaction to $80 \mathrm{ppm}$ of $\mathrm{NO}_{2}$ has been detected, whereas the structure $\mathrm{ZnO} \mathrm{B}$ gives then an audible response. It should be mentioned that in a dry mixture of $\mathrm{ZnO}$ the time of response (cf. Table) differs during the sorption and desorption only slightly (in the case of $\mathrm{ZnO} \mathrm{B}$ they are less than in the case of $\mathrm{ZnO} \mathrm{A}$ ).

The observed differences are probably due to the diametrically differing morphologies of the investigated $\mathrm{ZnO}$ structures. Comparing the response times of $\mathrm{TiO}_{2}$ and $\mathrm{ZnO}$, we find that the latter ones are slightly shorter. A higher concentration of humidity results in the case of $\mathrm{TiO}_{2}$ in a shortening of the response time during the sorption. In the course of desorption, for the case of $\mathrm{TiO}_{2}$ the time response is rather the same in dry and humid conditions. It would be difficult to quote a similar comparison concerning the behavior of such structures of $\mathrm{ZnO}$ in dry conditions, because the high threshold of detectability and the problem with only obey series of measurements provide only poor information. Basing, however, on the available information we may conclude that a large share of humidity leads to an increase of the values of changes of the resistance without increase of the threshold of detectability and does affect the response time. Difference in the characteristics resulting from the presence of various concentrations of humidity are probably due to the presence of more $\mathrm{OH}^{-}$ions causing an increase of the effectiveness of slowing down the active centres by the adsorbed oxygen, which increases the exchange of particles in these centres. This may accelerate the adsorption of $\mathrm{NO}_{2}$ and increase changes of the electric resistance. Neither is it impossible that on the surface of the semiconductors nitric acid is formed, the affectivity of its forming depending on the level of humidity.

Summing up, various morphologies of the nanostructures bring about differences in their electric reaction to the interaction with gas. A higher concentration of humidity in the gas mixture arises problems with the possibility of scaling the structure (the response of humidity in the investigated atmosphere has also its advantages, because it accelerates the reaction of the structure to gas and increases the values of responses (changes of the electric resistance)). Hence the conclusion may be drawn that the level of humidity must be controlled and kept on the proper level in the course of measurements, because its changes lead to changes in the response of the sensor.

The investigated materials may be potentially applied in gas sensors. But further attempts ought to be made to accelerate the response, which might be achieved by increasing the temperature of operation.

\section{Acknowledgments}

M.Sc. Marcin Procek is a scholar of the "SWIFT (Scholarships Supporting Innovative Technology Forum)" project POKL.08.02.01-24-005/10 co-financed by the European Union within the European Social Fund.

The investigations were sponsored by the National Centre for Scientific Researches and Development (NCBiR) within the grant O R00 017912.

\section{References}

[1] W. Zeng, T.M. Liu, Physica B, Condens. Matter 405, 1345 (2010).

[2] Y.X. Yu, D.S. Xu, Appl. Catal. B, Environm. 73, 166 (2007).

[3] E. Maciak, Z. Opilski, T. Pustelny, M. Bednorz, J. Phys. IV: JP (France) 129, 131 (2005).

[4] W.W. Guo, T.M. Liu, L. Huang, Mater. Lett. 65, 3384 (2011).

[5] T. Pustelny, M. Procek, E. Maciak, A. Stolarczyk, S. Drewniak, M. Urbańczyk, M. Setkiewicz, K. Gut, Z. Opilski, Bull. Pol. Acad. Sci., Techn. Sci. 60 , 853 (2012).

[6] B. Pustelny, T. Pustelny, Acta Phys. Pol. A 116 , 383 (2009).

[7] N. Yamazoe, K. Suematsu, K. Shimanoe, Sensors Actuat. B 163, 128 (2012).

[8] T. Pustelny, M. Setkiewicz, S. Drewniak, E. Maciak, A. Stolarczyk, M. Procek, M. Urbanczyk, K. Gut, Z. Opilski, I. Pasternak, W. Strupinski, Acta Phys. Pol. A 122, 870 (2012).

[9] T. Pustelny, M. Setkiewicz, S. Drewniak, E. Maciak, A. Stolarczyk, M. Urbańczyk, M. Procek, K. Gut, Z. Opilski, I. Pasternak, W. Strupiński, Bull. Pol. Acad. Sci., Techn. Sci. 61, 293 (2013).

[10] T. Pustelny, K. Barczak, K. Gut, J. Wojcik, Opt. Appl. 34, 53 (2004).

[11] M.H. Seo, M. Yuasa, T. Kida, J.S. Huh, N. Yamazoe, K. Shimanoe, Sensors Actuat. B 154, 251 (2011).

[12] M.E. Franke, T.J. Koplin, U. Simon, Small 2, 36 (2006).

[13] N. Yamazoe, K. Shimanoe, Sensors Actuat. B 138, 100 (2009).

[14] V.I. Gaman, Russ. Phys. J. 51, 425 (2008).

[15] K. Gut, Opt. Appl. 42, 407 (2012). 